PROCEEDINGS OF THE

AMERICAN MATHEMATICAL SOCIETY

Volume 139, Number 5, May 2011, Pages 1865-1869

S 0002-9939(2010)10693-7

Article electronically published on November 23, 2010

\title{
THE SLAMAN-WEHNER THEOREM IN HIGHER RECURSION THEORY
}

\author{
NOAM GREENBERG, ANTONIO MONTALBÁN, AND THEODORE A. SLAMAN
}

(Communicated by Julia Knight)

\begin{abstract}
Slaman and Wehner have independently shown that there is a countable structure whose degree spectrum consists of the nonzero Turing degrees. We show that the analogue fails in the degrees of constructibility. While we do not settle the problem for the hyperdegrees, we show that every almost computable structure, in the sense of Kalimullin, has a copy computable from Kleene's $\mathcal{O}$.
\end{abstract}

\section{INTRODUCTION}

A central concern of computable model theory is the restriction that algebraic structure imposes on the information content of an object of study. One asks about a countable object, what information is coded intrinsically into this object, which cannot be avoided by passing to an isomorphic copy of the object? Given a countable structure $\mathcal{M}$, we define the degree spectrum of $\mathcal{M}$ to be

$$
\operatorname{Spec}(\mathcal{M})=\left\{X \in 2^{\omega}: \exists \mathcal{N} \cong \mathcal{M}\left(\mathcal{N} \leqslant_{\mathrm{T}} X\right)\right\},
$$

where we identify $\mathcal{N}$ with its atomic diagram. In the language of mass problems, $\operatorname{Spec}(\mathcal{M})$ is the problem of computing a copy of $\mathcal{M}$. Since $\operatorname{Spec}(\mathcal{M})$ is degreeinvariant, we often replace $\operatorname{Spec}(\mathcal{M})$ by the collection of Turing degrees of elements of $\operatorname{Spec}(\mathcal{M})$. One of the major aims of computable model theory is understanding which collections of Turing degrees can be the spectra of some countable structures. Much study has gone into this problem; we refer the reader to 3$]$ for more information. We remark that Knight [6] has shown that unless $\mathcal{M}$ is trivial, if $X \in \operatorname{Spec}(\mathcal{M})$, then $X$ is Turing equivalent to some copy of $\mathcal{M}$.

Richter [7] has shown that every cone can be a degree spectrum; that is, for any Turing degree $\mathbf{d}$, there is a structure whose inherent information content is exactly d. On the other hand, Slaman [8 and Wehner 9 have independently shown that the collection of all noncomputable sets can also be the spectrum of a countable structure; in other words, there is a structure which captures noncomputability.

Unlike the structures whose degree spectrum is a (nontrivial) cone, Slaman's and Wehner's structures are almost computable: the Lebesgue measure of their degree

Received by the editors May 12, 2010.

2010 Mathematics Subject Classification. Primary 03C57, 03D60; Secondary 03D45.

The first author's research was partially supported by the Marsden fund of New Zealand.

The second author's research was partially supported by NSF grant DMS-0901169.

The third author's research was partially supported by NSF award DMS-1001551 and by the John Templeton Foundation.

(C) 2010 American Mathematical Society 
spectrum is 1. (Since the degree spectrum is $\Sigma_{1}^{1}(\mathcal{M})$, it is measurable; since it is invariant under Turing equivalence, it is either null or co-null.) The notion of almost computable structures was defined and investigated by Kalimullin [4] and Csima and Kalimullin [1, who in particular show that there is an almost computable structure whose degree spectrum is not co-countable.

In this paper we give an upper bound on the possible complexity of almost computable structures:

Theorem 1.1. If $\mathcal{M}$ is an almost computable structure, then there is some copy of $\mathcal{M}$ which is computable from Kleene's $\mathcal{O}$.

Here Kleene's $\mathcal{O}$ is the standard complete $\Pi_{1}^{1}$ set of natural numbers. We remark that Nies and Kalimullin (see [5]) have recently announced an independent proof of Theorem 1.1 indeed, they announced that if $\mathcal{M}$ is almost computable, then every $\Pi_{1}^{1}$-random set computes a copy of $\mathcal{M}$; Theorem 1.1 follows, because Kleene's $\mathcal{O}$ computes a $\Pi_{1}^{1}$-random set. Nies later showed that our result does imply his stronger one; we give details below.

Corollary 1.2. There are only countably many almost computable structures.

We note that in Theorem 1.1, Kleene's $\mathcal{O}$ cannot be replaced by any hyperarithmetic set. This is because it follows from results in [2] that for any computable ordinal $\alpha$, there is a countable structure $\mathcal{M}_{\alpha}$ whose degree spectrum consists of all sets $X$ such that $\Delta_{\alpha+1}^{0}(X) \nsubseteq \Delta_{\alpha+1}^{0}$. This spectrum is co-countable, and so $\mathcal{M}_{\alpha}$ is almost computable. On the other hand, given a computable ordinal $\beta$, we let $\alpha=\beta \cdot \omega$, so $\alpha+\beta=\alpha$, and so $\Delta_{\alpha+1}^{0}(\emptyset(\beta))=\Delta_{\beta+\alpha+1}^{0}=\Delta_{\alpha+1}^{0}$ and so $\emptyset^{(\beta)} \notin \operatorname{Spec}\left(\mathcal{M}_{\alpha}\right)$.

Hence no hyperarithmetic set can serve as a bound for all almost complete sets. We do not know though how sharp the bound given by Nies's and Kalimullin's result is. We have evidence supporting the fact that there is an almost computable structure with no hyperarithmetic copy, in fact one whose degree spectrum consists precisely of the nonhyperarithmetic degrees.

Theorem 1.1 motivates the following question: does the Slaman-Wehner theorem hold if we replace Turing reducibility by higher reducibilities? As mentioned above, we have reasons to believe that the answer is affirmative for hyperarithmetic reducibility, in a very strong way.

If we go further up in the hierarchy of reducibilities given by higher recursion theory, we arrive at relative constructibility. To make the situation nontrivial, the standard assumption on the underlying set-theoretic universe is that $\omega_{1}$ is inaccessible from reals, namely that for all $X \in 2^{\omega}, \omega_{1}^{L[X]}<\omega_{1}$ (equivalently, for all $X \in 2^{\omega}, \omega_{1}$ is inaccessible in $\left.L[X]\right)$. Under this assumption, we show that the Slaman-Wehner theorem fails for relative constructibility.

Theorem 1.3. Suppose that $\omega_{1}$ is inaccessible from reals. Then there is no countable structure $\mathcal{M}$ such that for all $X \in 2^{\omega}, L[X]$ contains a copy of $\mathcal{M}$ if and only if $X$ is not constructible.

\section{Almost computable structures}

In this section we prove Theorem [1.1 if $\mathcal{M}$ is a countable model such that $\lambda \operatorname{Spec}(\mathcal{M})>0$, then $\mathcal{M}$ has a copy computable from Kleene's $\mathcal{O}$ (here $\lambda$ denotes Lebesgue measure on $\left.2^{\omega}\right)$. Fix such a structure $\mathcal{M}$. 
Lemma 2.1. There is a partial computable function $\Phi: 2^{\omega} \rightarrow 2^{\omega}$ such that

$$
\lambda\left\{X \in 2^{\omega}: \Phi(X) \cong \mathcal{M}\right\}>1 / 2 .
$$

Proof. For a partial computable function $\Psi$, let

$$
C_{\Psi}=\left\{X \in 2^{\omega}: \Psi(X) \cong \mathcal{M}\right\} .
$$

There are only countably many partial computable functions, and the union of $C_{\Psi}$ for all partial computable functions is

$$
\left\{X \in 2^{\omega}: \exists \mathcal{N} \leqslant_{T} X(\mathcal{N} \cong \mathcal{M})\right\},
$$

which by assumption has measure 1 . Hence there is a partial computable function $\Psi$ such that $\lambda C_{\Psi}>0$. By the Lebesgue density theorem, there is some finite string $\sigma \in 2^{<\omega}$ such that

$$
\frac{\lambda\left(C_{\Psi} \cap[\sigma]\right)}{2^{-|\sigma|}}>1 / 2
$$

We let $\Phi(X)=\Psi(\sigma X)$.

We fix a partial computable function $\Phi$ given by Lemma 2.1 and let

$$
C=C_{\Phi}=\left\{X \in 2^{\omega}: \Phi(X) \cong \mathcal{M}\right\} .
$$

Let

$$
A=\left\{(X, Y) \in\left(2^{\omega}\right)^{2}: \Phi(X) \cong \Phi(Y)\right\} .
$$

Then $A$ is a $\Sigma_{1}^{1}$ class. For any $X \in 2^{\omega}$, let $A_{X}$ be the section

$$
A_{X}=\left\{Y \in 2^{\omega}:(X, Y) \in A\right\} .
$$

Fix some rational number $q>1 / 2$ such that $\lambda C \geqslant q$. Let

$$
B=\left\{X \in 2^{\omega}: \lambda A_{X} \geqslant q\right\} .
$$

Lemma 2.2. $B$ is a $\Sigma_{1}^{1}$ class.

Proof. There is a computable function $\Psi:\left(2^{\omega}\right)^{2} \rightarrow 2^{\omega}$ such that for all $X$ and $Y$, $\Psi(X, Y)$ is a linear ordering of $\omega$ and $(X, Y) \in A$ iff $\Psi(X, Y)$ is not well-founded. For $\alpha<\omega_{1}$, we let $A_{\alpha}$ be the set of pairs $(X, Y)$ such that $\Psi(X, Y)$ is not embeddable into $\alpha$.

By Spector's argument, for all $X, \lambda A_{X}=\lambda\left(A_{\omega_{1}^{X}}\right)_{X}$, so $X \in B$ if and only if for all $\alpha<\omega_{1}^{X}, \lambda\left(A_{\alpha}\right)_{X} \geqslant q$. Now, we have a computable function $\Xi$ which, given $X$ and a notation in $\mathcal{O}^{X}$ for an ordinal $\alpha<\omega_{1}^{X}$, gives a $\Delta_{1}^{1}(X)$-index for $\lambda\left(A_{\alpha}\right)_{X}$, since $\left(A_{\alpha}\right)_{X}$ is $\Delta_{1}^{1}(X)$, uniformly in $X$ and $\alpha$.

Hence

$$
X \in B \Leftrightarrow \forall n\left(n \in \mathcal{O}^{X} \rightarrow q \leqslant \Xi(n, X)\right),
$$

which is a $\Sigma_{1}^{1}$ definition of $B$.

Lemma 2.3. $B=C$.

Proof. If $X \in C$, then for all $Y \in C,(X, Y) \in A$, so $C \subseteq A_{X}$, so $\lambda A_{X} \geqslant q$, so $X \in B$

Suppose that $X \in B$. Since $\lambda A_{X}, \lambda C>1 / 2$, the intersection $A_{X} \cap C$ is nonempty; let $Y \in A_{X} \cap C$. Then $\Phi(Y) \cong \mathcal{M}$ since $Y \in C$, and $\Phi(Y) \cong \Phi(X)$ since $Y \in A_{X}$. Hence $\Phi(X) \cong M$, so $X \in C$. 
Now Theorem 1.1 follows from the basis theorem for $\Sigma_{1}^{1}$ classes: every nonempty $\Sigma_{1}^{1}$ class contains a set computable from Kleene's $\mathcal{O}$.

As mentioned above, Nies later observed that our result implies his stronger version: that if $\mathcal{M}$ is an almost computable structure, then every $\Pi_{1}^{1}$-random set computes a copy of $\mathcal{M}$. We have established that for any almost computable structure $\mathcal{M}$, there is a nonnull $\Sigma_{1}^{1}$ class $B$ such that every $X \in B$ computes a copy of $\mathcal{M}$. The Nies-Kalimullin result follows from the following observation of Nies's:

Proposition 2.4. Let $B$ be a $\Sigma_{1}^{1}$ class of positive measure. Then for any $\Pi_{1}^{1}$ random set $X$, there is some $Y \equiv_{\mathrm{T}} X$ in $B$.

Proof. We in fact show that $B$ contains a tail of $X$. Recall that for all $\sigma \in 2^{<\omega}$,

$$
C \uparrow_{\sigma}=\left\{Z \in 2^{\omega}: \sigma Z \in B\right\} ;
$$

we have $\left.\lambda B\right|_{\sigma}=2^{|\sigma|} \lambda(B \cap[\sigma])$. Let

$$
D=\bigcup_{\sigma \in 2^{<\omega}} B \uparrow_{\sigma} .
$$

Since the sets $\left\langle\left. B\right|_{\sigma}\right\rangle$ are uniformly $\Sigma_{1}^{1}, D$ is also $\Sigma_{1}^{1}$. By the Lebesgue density theorem, since $\lambda B>0$, we have $\lambda D=1$. Since $X$ is $\Pi_{1}^{1}$-random, $X \in D$, so some tail of $X$ is in $B$.

\section{The CONSTRUCtible SPECTRUM}

We adapt the argument of the previous section to prove Theorem 1.3 . We need to relativise the proof to a countable ordinal $\alpha$; but as we now show, by restricting to a co-null class, we may assume that $\alpha$ is countable in $L$.

Let $\mathcal{M}$ be a countable structure, and suppose that for any nonconstructible $X \in 2^{\omega}, L[X]$ contains some copy of $\mathcal{M}$. We assume that $\omega_{1}$ is inaccessible from reals; so for all $X \in 2^{\omega}, \omega_{1}^{L[X]}$ is countable, and so $2^{\omega} \cap L[X]$ is countable.

Since $L$ contains only countably many Borel codes, the collection of $X \in 2^{\omega}$ which are random over $L$ is co-null. Since random real forcing does not collapse $\omega_{1}$, for each $X$ which is random over $L$, we have $\omega_{1}^{L[X]}=\omega_{1}^{L}$. Hence, the collection

is co-null.

$$
N=\left\{X \in 2^{\omega}: \omega_{1}^{L[X]}=\omega_{1}^{L}\right\}
$$

For all $X \in 2^{\omega}$, fix a uniformly $\Delta_{1}^{L[X]}$ bijection $j_{X}$ from $\omega_{1}^{L[X]}$ to $2^{\omega} \cap L[X]$.

Lemma 3.1. For any $\alpha<\omega_{1}$, the relation $Y=j_{X}(\alpha)$ is Borel.

Indeed, the relation is $\Delta_{1}^{1}(R)$ for any real code $R$ for $\alpha$.

Proof. Let $R \in 2^{\omega}$ be a code for $\alpha$. Then $Y=j_{X}(\alpha)$ if and only if for some (for all) $\omega$-models $M$ of $\mathrm{ZFC}^{-}$that contain $R, X$ and $Y, M=" Y=j_{X}(\alpha)$ ".

For $X \in N \backslash L$, there is some $\alpha<\omega_{1}^{L}$ such that $j_{X}(\alpha)$ is isomorphic to $\mathcal{M}$. By Lemma 3.1, for any $\alpha<\omega_{1}^{L}$, the class

$$
K_{\alpha}=\left\{X \in 2^{\omega}: j_{X}(\alpha) \cong \mathcal{M}\right\}
$$

is $\Sigma_{1}^{1}$ and so is measurable. Since $\omega_{1}^{L}$ is countable and $N \backslash L$ is co-null, there is some $\alpha<\omega_{1}^{L}$ such that $\lambda K_{\alpha}>0$. Again by Lebesgue density, there is some finite $\sigma \in 2^{<\omega}$ such that

$$
C=\left\{X \in 2^{\omega}: \sigma X \in K_{\alpha}\right\}
$$


has measure strictly greater than $1 / 2$. For all $X \in 2^{\omega}$, let $\Phi(X)=j_{\sigma X}(\alpha)$.

Fix some $R \in L \cap 2^{\omega}$ which is a code for $\alpha$. Let

$$
A=\left\{(X, Y) \in\left(2^{\omega}\right)^{2}: \Phi(X) \cong \Phi(Y)\right\} .
$$

Lemma 3.1 implies that $A$ is $\Sigma_{1}^{1}(R)$. We again fix some rational $q>1 / 2$ such that $\lambda C \geqslant q$, and let

$$
B=\left\{X \in 2^{\omega}: \lambda A_{X} \geqslant q\right\} .
$$

The argument of Lemma 2.2 shows that $B$ is $\Sigma_{1}^{1}(R)$, and the argument of Lemma 2.3 shows that $B=C$. Hence there is a copy of $\mathcal{M}$ constructible from $\mathcal{O}^{R}$; since $R \in L$, we have $\mathcal{O}^{R} \in L$, and so $\mathcal{M}$ has a constructible copy as required.

\section{REFERENCES}

[1] Barbara Csima and Iskander Sh. Kalimullin. Degree spectra and immunity properties. Math. Logic Quarterly, 56(1):67-77, January 2010. MR2598838

[2] Sergey S. Goncharov, Valentina S. Harizanov, Julia F. Knight, Charles McCoy, Russell Miller, and D. Reed Solomon. Enumerations in computable structure theory. Ann. Pure Appl. Logic, 136(3):219-246, 2005. MR2169684 (2006f:03071)

[3] Valentina S. Harizanov. Pure computable model theory. In Handbook of recursive mathematics, Vol. 1, volume 138 of Stud. Logic Found. Math., pages 3-114. North-Holland, Amsterdam, 1998. MR1673621(2000f:03108)

[4] Iskander Sh. Kalimullin. Some notes on degree spectra of structures. In S. Barry Cooper, Benedikt Löwe, and Andrea Sorbi, editors, Computation and Logic in the Real World, volume 4497 of Lecture Notes in Computer Science, pages 389-397. Computability in Europe, Springer, 2007.

[5] André O. Nies. Randomness and computability: five questions. To appear in Studying randomness through computation, H. Zenil (ed.), World Scientific.

[6] Julia F. Knight. Degrees coded in jumps of orderings. J. Symbolic Logic, 51:1034-1042, 1986. MR865929 (88j:03030)

[7] Linda J. Richter. Degrees of structures. J. Symbolic Logic, 46(4):723-731, 1981. MR641486 (83d:03048)

[8] Theodore A. Slaman. Relative to any nonrecursive set. Proc. Amer. Math. Soc., 126(7):21172122, 1998. MR.1443408 (98h:03047)

[9] Stephan Wehner. Enumerations, countable structures and Turing degrees. Proc. Amer. Math. Soc., 126(7):2131-2139, 1998. MR.1443415 (98h:03059)

School of Mathematics, Statistics and Operations Research, Victoria University, Wellington, New Zealand

E-mail address: greenberg@msor.vuw.ac.nz

Department of Mathematics, University of Chicago, 5734 S. University Avenue, Chicago, Illinois 60637

E-mail address: antonio@math.uchicago.edu

Department of Mathematics, University of California Berkeley, Berkeley, CaliforNIA $94720-3840$

E-mail address: slaman@math.berkeley.edu 